\title{
NOTE SUR LE REPEUPLEMENT ARTIFICIEL DU BROCHET
}

\author{
par P. CHIMITS \\ Ingénieur agronome \\ Inspecteur des Eaux et Forêts \\ Chef de la $1^{\text {te }}$ Région piscicole
}

Le Comilé Central de la Pêche nous a voté, le 15 Février 1946 , une subvention de 30.000 francs, et le 20 Juin I946, une autre de 6o.0oo francs pour recherches sur l'élevage du Brochet. Les pêcheurs, en effet, depuis quelques amněes, attiraient lattention du Service de la Peche sur le dépeuplement de nos rivières en Brochet. Il n'est plus besoin d'insister sur la nécessité, admise à présent par tous, de la présence d'une certaine proportion de voraces, et surtout de Brochets, dans nos rivières, pour contenir la pullulation de la blanchaille et maintenir l'équilibre de nos eaux.

Nous rendons compte des résultats de nos travaux depuis deux ans et qui, nous l'espérons, vont aboutir à des résultats concrets, puisque nous pouvons, dès à présent, annoncer que nous présentons un projet de construction à l'étang du Der (Haute-Marne), d'une pisciculture capable de traiter 5 millions d'oufs de Brochet qui seront fournis par les géniteurs de cet étang et qui pourra, nous l'espérons, fonctionner dès s948. Nous avons, dès cette année, obtenu 800.000 oufs de Brochet au Der. Mais ce résultat n'a pas été obtenu sans des déboires et certains échecs préalables.

Dès nos premières recherches, en I 945 , nous avons pu vérifier que la méthode de fécondation et d'incubation employèe par les Suisses, notamment Vougis, et les Allemands était parfaitement au point.

L'ouvrage de Vouga sur l'ésociculture, publié en 1938, indique thous les détails pratiques de la technique de l'élevage en carafe de Zoug. Gràce à cet appareil et en suivant les indications de Vouga, il est facile, si on a la chance de tomber sur une femelle de Brochet bien mùre et des máles féconds, d'opérer la fécondation artificielle et d'obtenir des oufs embryonnés et des alevins de Brochet. M. WuRTz, Chef de Travaux à la Station Centrale d'Hydrobiologie appliquée, a donné la description très détailléa de l'évolution de l'euf et de l'alevin de Brochet dans le numéro i $35 \mathrm{du}$ Bulletin Français de Pisciculture. (Octobre-Décembre 1944).

Vouga préconise la méthode extra sèche et indique les soins minutieux 
qu'il faut prendre pour nettoyer les géniteurs de leurs mucus et éliminer toute trace d'eau dans la cuvette de fécondation.

Nous estimons, toutefois, qu'il n'y a pas lieu d'exagérer ces précautions. Nous avons vu opérer les pisciculteurs allemands du lac de Constance qui n'essuient ni le récipient, ni les géniteurs, et n'attachent aucune importance au jef d'eau qui précède sowent la chute des ovules. Je garde-pèche Ducoffe a, d'ailleurs, opéré à l'établissement de Reichenau, près Constance,

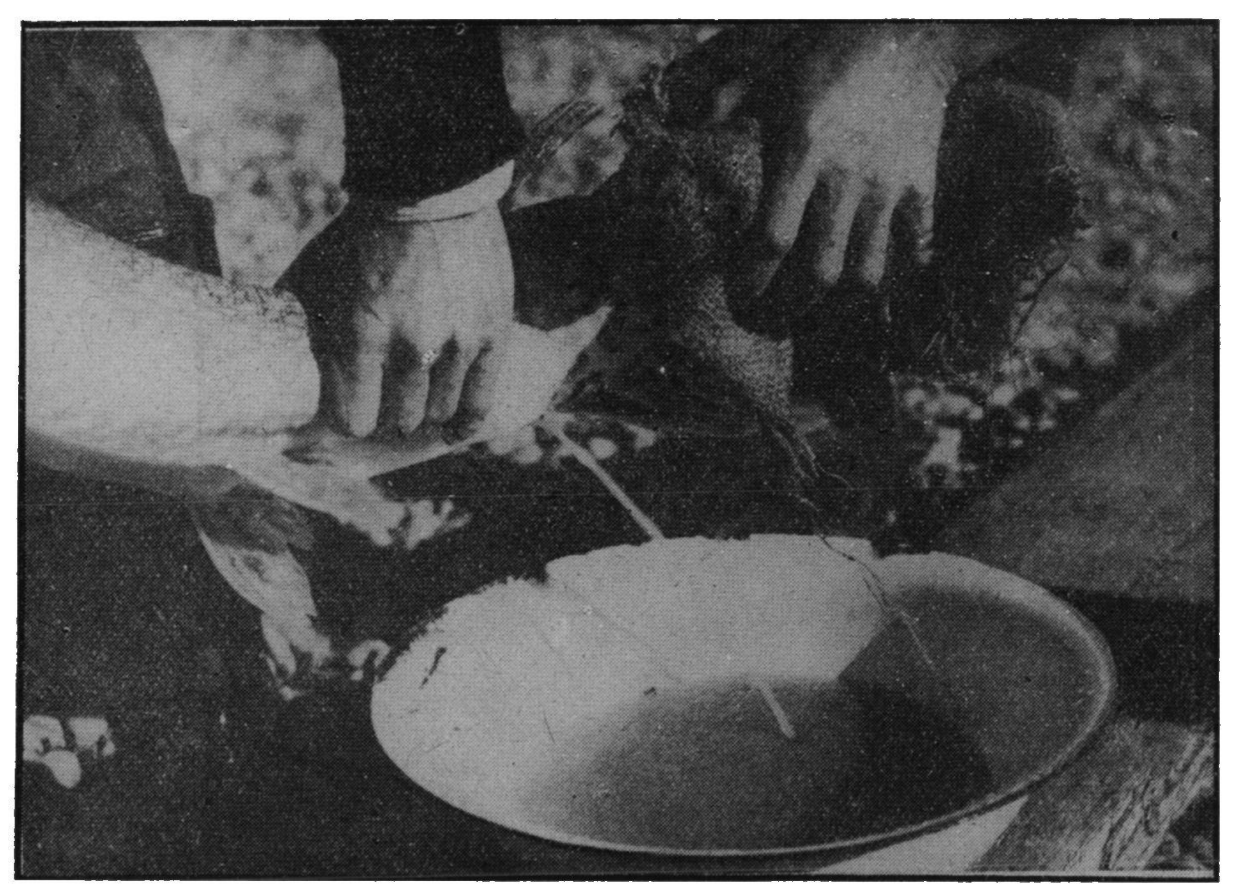

Fıc. '. - Fécondation artificielle du Brochet par la méthode sèche.

une fécondation extra sèche dont la réussite a été la mème que celle ur's fécondations semi-humides des Nllemands.

S'il est bon d'arroser les coufs d une ou deux femelles de la laitance de plusieurs mâles, celà n'est pas nécessaire si le nombre des mâles est insuffrsant. En cas de nécessité, la laitance d'un seul mảle peut être suffisante.

Nous ajouterons que les œufs ainsi fécondés, comme des oufs de Truite, et lavés une seule fós, preuvent ìtre Iransportés dạns l'eau pour n'être mis qu'une ou deux heures plus tard en carafe de 7oug.

En résumé, la fécondation se fait par voie sèrhe (Fig. 4), ef l’incubation en carafe (Fig. i) permet d'ohtenir l'éclosion, dix à douze jours après, de $70 \%$ des cufs.

Dès r $945 \overline{5}$, nous avons obtenu l'éclosion de 20.000 aufs de Brochet à l'Etablissement de Neuville, et nous avons pu constater que les difficultés 
pour obtenir des résultats suffisanment importạnts pour entreprendre une campagne de réempoissonnement şstématique de nos rivières en Brochet, se situaient :

$1^{\circ}$ Dans la maturation des génileurs ;

$2^{\circ}$ Dans la méthode de réempoisssonnemenl, une fois arrivé au stade de l'oruf fécondé et embryonné.

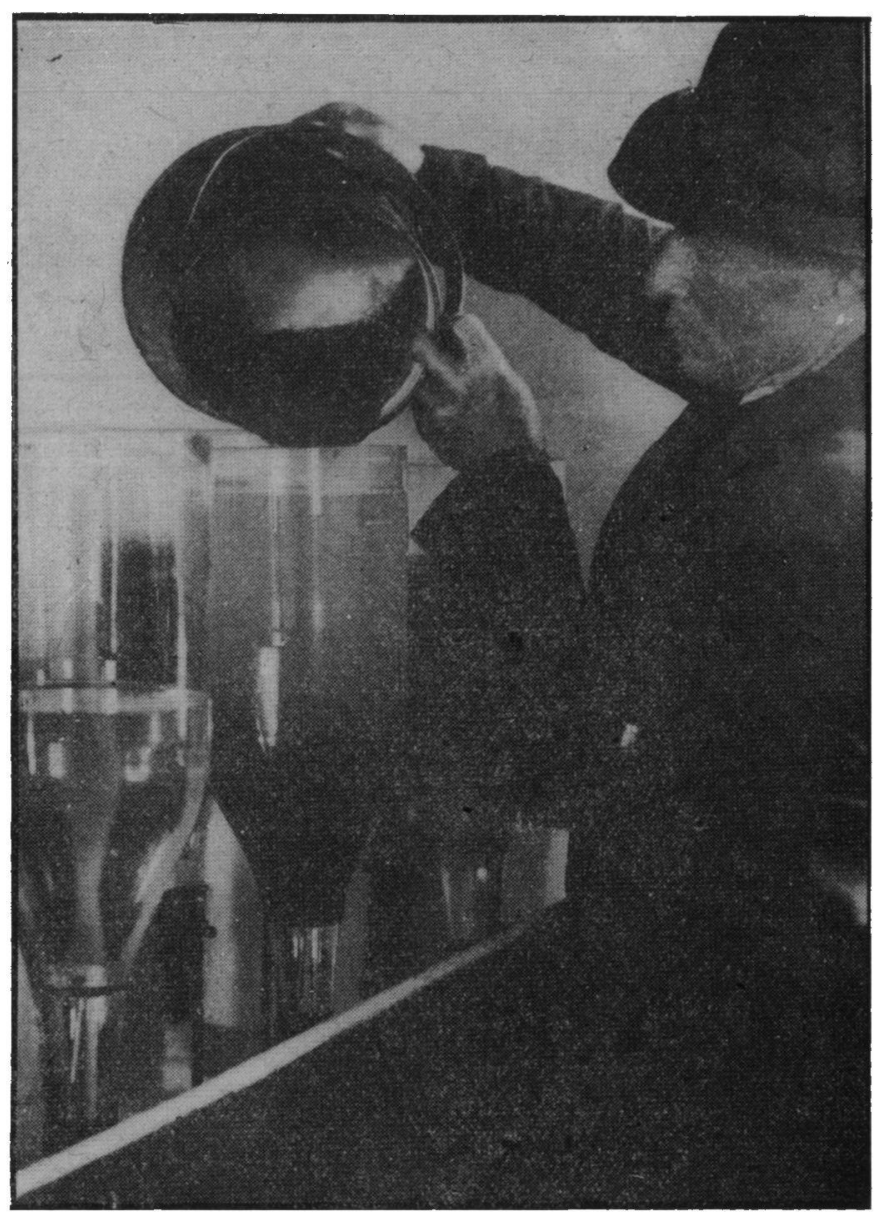

Fıg. 5. - Remplissuge de lia carafe de Zoug+

En eflet, pour l'ésociculture industrielle, nous citerons Vovga : " En Suisse, en ig36, les bruchetons déversés n'entrent en ligne de compte que pour 20.500, c'est-à-dire que cet élevage n'est pas à la portée de tout le monde, et qu'il ne peut àtre entrepris que si l'on se trouve dans des conditions exceptionnellement favorables ").

Ceci dit, nous sommes d'accord avec $M$. Wuntz qui déclare n'avoir 
trouvé aucune difficulté spéciale dans l'ésociculture, mais à condition de considérer le terne d'ésociculture dans le seps de lobtention, en laboratoire, de quelques alevins de Brochet. Autre chose est l'ésociculture industrielle où les deux problèmes indiqués ci-dessus, et que nous allons examiner, n'étaient pas, à notre connaissance, résolus en ce qui concerne notre domaine piscicole national.

De la solution de ces deux problèmes pratiques, dépond la mise en roule de l'ésociculture française. Nous pensons les avoir résolus.

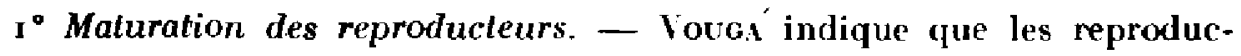
teurs doivent être capturés sur les frayères et fécondés dẻs que possible.

Au cours de notre voyage d'études, en 19/7, au Lac de Constance, nous avons assisté avec le Dr. Kocn, Chef du Service de la Pisciculture au Lac de Constance, atux peches des reproducleurs par les pecheurs professionnels. Les Brochets sont amenés dans les boutiques à poissons chez le marchand de poissons en gros où le prisciculteur vient procéder au prólèvement et à la fécondation artificielle des ouls cui sont cusuite placés dans les carales de Zoug de l'établissement d'ésociculture voisin de Reichrnau.

Le Dr. Косн nous a précisé qu'il n'avait jamais pu obtenir de matıration sexuelle chez des Brochets captifs. Nous an ćlions convaincus, deux années d'expériences décevantes à Neuville nous ayant prouvé que lorsque le Brochet ne donne pas ses oufs au moment de sa capture, il se refuse à mûrir en captivité. Ni les injections de produits ovariens (tels que benzo cestradiol, par exemple), efficaces pourtant rhey les animaux à sangr (lhaud, ni la mise en place des gréniteurs dans un bac chauffant construit spécialement à cet effet, afin de les maintenir dars une cau à ${ } i^{\circ}$, favorable à la maturation, ne nous ont dọné le moindre résultat.

Or il n'y a pas, en France. de lacs on de rivières où les pioheurs professionnels soient assez nombreux ot assez disciplines pour apporter des Brochets mûrs vivants a u pisciculteur en quantité suffisante.

Nos ésocicultures ne pouvaient done fournir que quelques dizaines de: milliers d'ceufs par an. par cotte méthode.

Une observation faite au mois de Mars rg/7 à l'élang du Der, nous a donné la possibilité de capturer très facilement des géniteurs Brochets sexuelilement mûrs.

L'étang du Der, d'une superficie de joo hertares, et rue nous sommes en train d'aménager. contient de nombreux Brochets. Nous avons, l'automne 1946 , clos une des deux queues de cet étang où se déverse le ruisseau de la Droye, afin de le transformer en un étang de grossissement de 2o hectares, par une grille à mailles fines, par laquelle se déverse un léger courant d'eau.

Dès le début de Mars, nous avions constaté une migration massive de Brochets qui s'accumulaient le nez contre le grillage. Ces Brochets res. taient immobiles, et grảce à un petit carrelet de so centimètres de côté, 
il était facile d'en capturer cinq ou six à chaque coup (Fig. 6). Ces Brochets étaient tous sexuellement mùrs.

Avec des moyens de fortune, nous avons mis en place deux carafes de Zoug et prélevé, dans le même après-midi, 800.000 œufs. Etant donné qu'il se pressait à la grille des centaines, voire des milliers de Brochets, nous estimons que si nous avions eu des installations de carafés nécessaires, nous aurions pu préleverr, en quelques jours, $i$ à 6 millions d'wufs.

La migration a duré truis semaines environ.

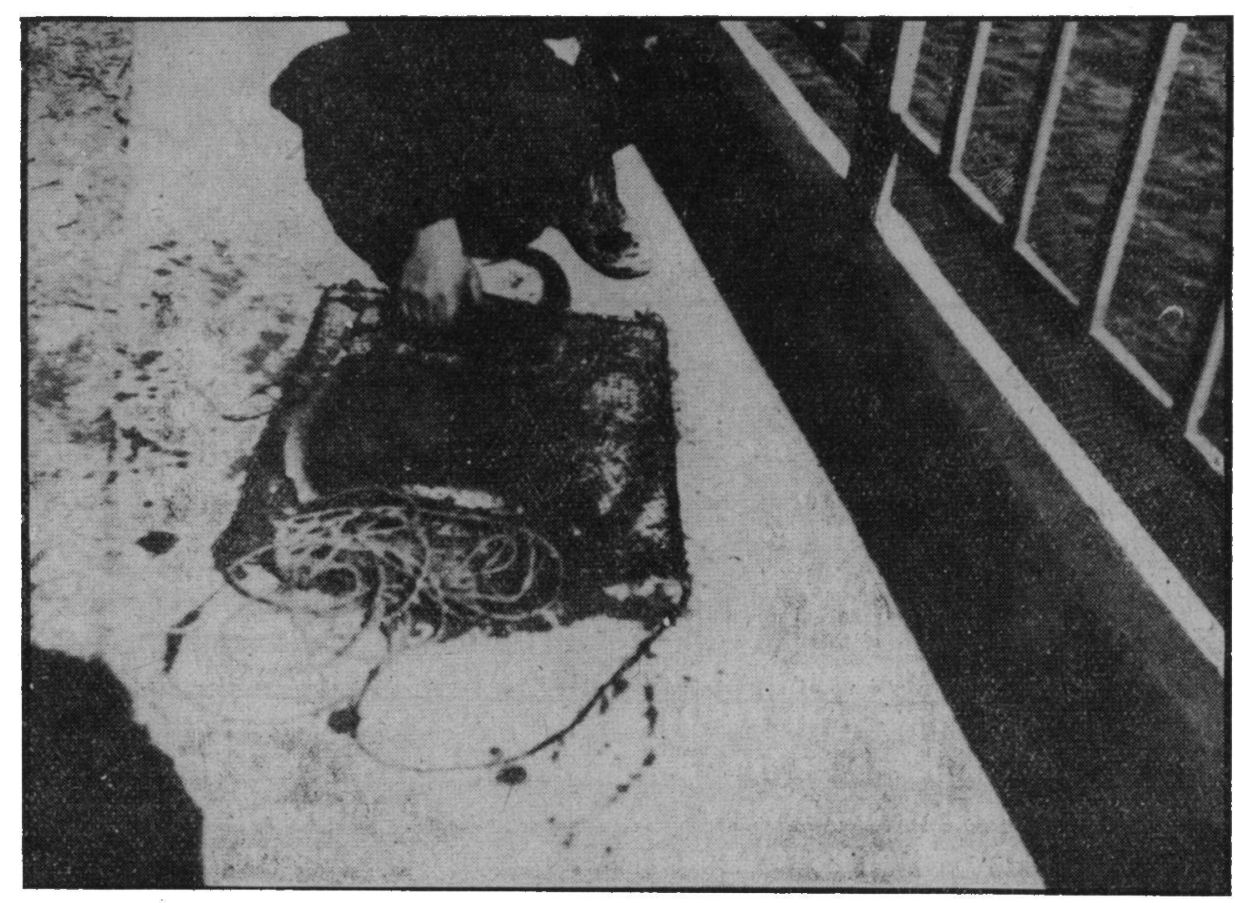

Fig. . 6- Capture des géniteurs de Brochet au carrelet devant la grille.

Une courte expérience nous a permis, en ouvant le canal de remplissage du Der, à y kilomètre environ de la triille, de disperser la masse de Brochets. Nous avons ensuite fermé le canal de remplissage et le lendemain, les Brochets se rassemblaient à nouveau à l'appel d'cau de notre grille.

En résumé, pár un simple système d'arrivée d'eau à une température convenant à la fraye, on peut attirer et capturer très facilement les Brochets sexuellement mûrs d'une nappe d'eau. Les conditions se prêtent, au Der, à l'établissement d'une ésociculture pouvant traiter plusieurs millions d'œufs. Une telle station de capture semble pouvoir être créée dans d'autres lacs, tels que les lacs des Landes, par exemple. Mais il nous semble nécessaire d'étudier de près ce phénomène de la migration des Brochets, et de 
noter de façon précise les qualités de l'eau qui les attire. A première vue, nous ne voyons pas d'autres raisons que le courant et peut-être la température un peu plus tiède.

$2^{\circ}$ Méthodes de réempoissonnement en Brochets. - Vouga a montré l'impossibilité pratique de l'élevage du brocheton à l'état sevré, en raison du cannibalisme instinctif de l'alevin dès la troisième semaine de l'éclosion, quelle que soit la quantité de nourriture, même vivante, qui lui est offerte. Il est nécessaire, à son avis, de disposer de très grands étangs peu profonds et riches en nourriture naturelle.

L'élevage, dans la même auge, de quelques milliers d'alevịns arrive à la production, en quelques semaines, d'un seul brocheton qui finit par avoir dévoré tous ses congénères.

Vouga pratique donc en Suisse la répartition, sur de grandes étendues, de très jeunes alevins et surtout d'cufs embryonnés sorlis de la carafe de Zoug, le jour ou la veille du jour de l'éclosion.

Ies Allemands, au lac de Constance, font édore les oufs de Brochet et gardent les alevins jusqu’a la résorption de la vésicule, soit deux semaines après l'éclosion. Ils les déversent dans le lac qui touche à l'établissement.

Il n'est pas possible, en France, d'élever systématiquement en étang les brochetons d'un an, élevage qui ne donnerait, le plus souvent, que des déboires, et ne peut constituer qu'un produit aléatoire.

Quant aux lalevins de 14 jours, à la résorption de la vésicule, ils sont pratiquement intransportables à certaine distance et ne peuvent servir qu'à l'alevinage des grands lacs par les piscicultures voisines.

La seule méthode qui nous a paru applicable à des réempoissonnements sur de longues étendues de rivière, est celle de la répartition sur les herbes aquatiques d'oufs embryonnés de Brochets près de l'éclosion, c'est-à-dire entre le septjème et le onzième jour d'incubation dans les carafes' de Zoug (Fig. 7).

Cesiøufs sont transportés, à courte distance, dans des récipients garnis d'eau ; à longue distance, comme des ceufs de Truite, dans des clayettes de toile entourées de mousse humide et de glace. Ils sont déposés sur les herbes aquatiques a l'aide d'une plume, par groupe de 3 ou 4 , en séparant chaque groupe d'une distance de 5 mètres. Ainsi les jeunes alevins éclos trouvent-ils de suite leur espace vital et ne s'entre dévorent-ils pas.

Cette méthode est pratiquée en Suisse, en eau libre, mais nous n'avons pu trouver de pourcdrtage de réussite de ces déversements d'œufs.

Résultats de la méthode. - Afin de donner aux pêcheurs une indication sur le nombre d'œufs de Brochet à déverser au kilomètre de rivière, nous avons, depuis 1945 , procédé à des expériences de déversement en eau close, à Neuville et à Courville. Ci-dessous quelques résultats :

Etang III, de Neuville. - 2 hectares. - Mise an place, le ro Mars 1945 de 
2.000 cufs embryonnés de ro jours (volume $33 \mathrm{~cm}^{3}$ ). - Cet étang contenait, outre des Carpes, Tanches et Gardons, i 4 brochetons de 80 à roo grammes, non mûrs, et qui ont dû dévorer une parlia de nos alevins de Brochet. Les conditions étaient donc analogues à celles d'une rivière normale.

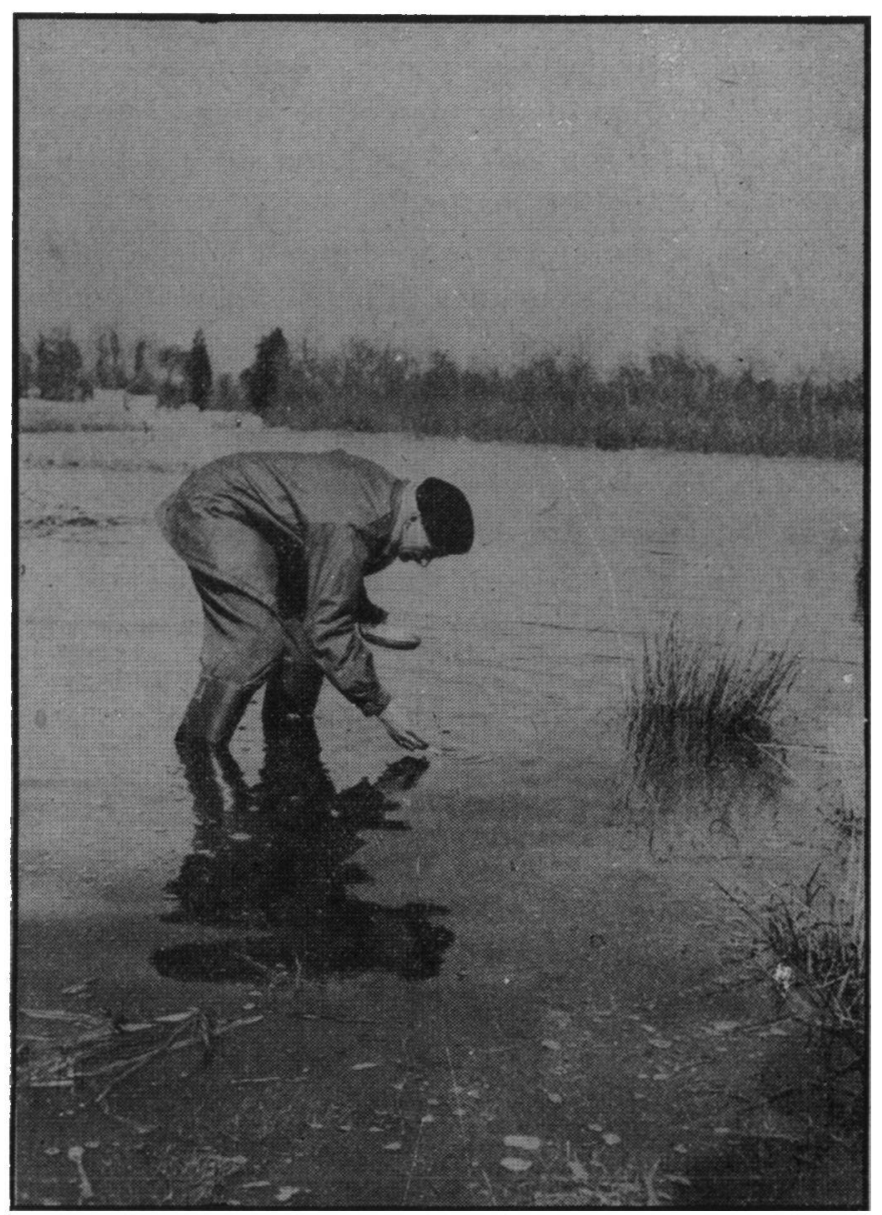

Fig. 7. - Déversement en étang des ceuts embryonnés de Brochet.

Le 23 Octobre 1945 , sept mois plus tard, nous retrouvions i3 Brochets de deux étés qui étaient passés d'un poids moyen de 100 grammes à un poids moyen de I $\mathrm{kg}_{\mathrm{g}} \mathbf{2}^{2}$, et $6 \mathrm{I}$ Brochets de sept mois, d'un poids total de 19 kilogs, soit 3 Io grammes en moyenne chacun, pour une taille allant de 27 à 38 centimètres.

Etang II, de Courville. - o hectare 53. - Mise en place le 12 Avril 1946, de 500 cufs $\left(\mathrm{Io}^{3} \mathrm{~cm}^{3}\right)$ de io jours. - Pêche le 26 Octobre 1946 de 55 brochetons d'un poids total de $6 \mathrm{kgs}$ o5o et d'un poids moyen de 110 grammes.

Etang I, de Neuville. - o hectara 50. - Mise en place le 12 Avril 1946 
de 500 ceuls (10 $\mathrm{cm}^{3}$ ) de ro jours. - L'étang contenait de grosses Truites communes géniteurs et des Gardons. - La pèche fin Octobre, a donné 3 r Brochets de 22 à 27 ceṇtimètres.

Etang II, de Neuville. - 2 hectares. - Mise en place, le 12 Avril 1946 , de 500 cufs $\left(10 \mathrm{~cm}^{3}\right)$ de ro jours. - Létangr contenait joo Truites arc-enciel de 14 mois, d'un poids de 80 à 100 grammes, des Tanches et des Gardons. - La pèche, en Mars 1947 , a donné 13 brochetons de 500 grammes de moyenne. Quant aux truitelles arc-en-ciel, elles avaient disparu (en passant, notons l'íncompatibilité de la Truite et du Brochet).

Etang III, de Neuville. - 2 hectares. - Miset en place, le I 2 Avril 1946, de joo oufs (ro $\mathrm{cm}^{3}$ ) de ro jours. - L Létang contenait des géniteurs de Gardons et de Tanches. - La pèche, à l'automne r946, a permis de retrouver 7 Brochets, dont 6 de 23 à 37 centimètres, et un de 75 centimètres. Ce dernier semble être un Brochet de deux ans non retrouvé lors de la pèche précédente.

Bassin de Saint-Gilles. - o hectare 25, très enherbé. - Mise en place, le 12 Avril 1946 , de r 25 oufs $\left(5 \mathrm{~cm}^{3}\right)$ de 10 jours. - La pêche, à l'automne, a permis de retrouver 2 Brochets de 130 grammes.

En résumé, les déversements d'oufs embryonnés à l'àge de ro jours ont donné, sept mois plus tard, de $1,4 \%$ à is $\%$ en nombre de brochetons de roo à 300 grammes, soit, en moyenne $5 \%$.

Approximativement, le déversement de r.ooo cufs embryonnés (soit $20 \mathrm{~cm}^{3} \mathrm{~d}$ 'cufs) dans un hectare d'eau (ou, par analogie, dans une rivière de I kilomètre de long et io mètres de large) permet d'espérer l'obtention, à l'automne suivant, de 50 brochetons de 100 à 200 grammes.

Cette densité de Brochet, dans une rivière de ro mètres de larợe, soit tu, lous les 20 mètres, semblant rationnelle, nous estimons, en attendant que de nouvelles expériences viennent apporter des précisions sur la question, qu un déversement de $x .000$ à 2.000 oufs embryonnés par kilomètre de rivière de 10 mètres de large, peut être rccommandé. Si la rivière est plus large, le nombre d'oufs à déverser doit.être, évidemment, plus grand et proportionnel à la largeur.

Transport des oufs. - Dès le septième jour de l'embryonnement, les cufs sont très facilement transportables à long̣ue distance, pendarit 24 ou 36 heures. On les transporte dans des clayettes entourées de mousse et de glace, comme des ceufs de Truite.

Arrivés au lieu de répartition, il convient de distribuer une clayette contenant de r $\overline{5}$ à 30.000 cufs à un seul déverseur qui se chargera de déposer les œufs sur un secteur déterminé de rivière.

Nous avons ainsi réussi à lransporter des aufs de Brochet du Der dans le Loiret (40o kilomètres), dans l'Aisne, etc...

Un essai de transport par avion, au Maroc, a été un échec, les ceufs, 
agés de I I jours, ayant éclos en cours de route. Mais un tel transport, avec des ouls àgés de 7 jours, ent été possible.

D'ailleurs, nous avons réussi à déverser, en bon état, dans le Haut-Rhin et le Bas-Bhin, des oufs de Brochet provenant du Lac de Constance, après un trajet de 24 heures.

Ces divers résultals nous permettent de proposer le projet ci-joint de repeuplement de nos rivièreg en Brochets.

\section{Projet de hepeuplement des hivì̀mes françatges en brochet}

$I^{\circ}$ Création, à l'étang du Der (Haute-Marne), d'une station centrale d'ésociculture qui permettra de récolter et d'incuber 5.000.000 d'œufs de Brochelt, et de desservir les Fédérations de la moitié Nord de la France.

$2^{\circ}$ Etude de la mise en place d'une station centrale analogue auprès d'un des grands étangs des Landes qui pourrait desservir la moitié Sud.

$3^{*}$ Mise en place, auprès de chaque Fédération désireuse d'aleviner en Brochet, de deux carafes de Zoug pour recevoir - des deux stations centrales - les ceufs de 7 jours et continuer leur évolution jusqu'à ro ou I I jours. Ce délai permettra la convocation, par fe Président de la Fédération, pour le $10^{\circ}$ ou $I^{*}$ jour, d'une equipe de volontaires munis d'autos et bicyclettes qui, une fois l'an, déposeront les oufs de Brochel en rivière selon la technique indiquée et le plan établi à l'avance, sur la base de 1.000 à 2.000 ceufs par kilomètre de rivière de 10 mètres de large ( 1 ).

$4^{\circ}$ Nous proposons, en outre, que les carpiculteurs déversent dans leurs étangs de trois étés, des ceufs embryonnés de Brochet. Les Carpes et les Tanches, dans leur troisième année, ne craignant rien des brochetons. Elles pèseront, en effet, qui trois livres, qui une livre à une livre et demie, alors que les brochetons ne feront qu'une demi-livre en moyenne.

En ce cas, naturellement, il conviendra le charger l'étang d'une trentaine de gros géniteurs Gardons à l'hectare, dont le frai servira de nourriture aux brochetons.

Ainsi les carpiculteurs bénéficieront-ils d'un produit de grande valeur, tant pour la vente à l'industrie de la quenelle, que pour la vente aux associations de Péche, sans risquer les dégâts que les brochetons d'un été déversés au printemps ne manquerajent pas de causer, avant l'automne, à leur chargement de Carpes et de Tanches.

(1) La création de telles petites stations secondaires de carafes de Zoug n'est d'ailleurs pas nécessaire si les ceufs sont déversés en rivière dans les 24 heures de la réception. 